\title{
Social Network Dynamic Modeling via Mathematical Applicative Formulae
}

\author{
Isabelle Pope \\ Wageningen University \& Research \\ Netherlands
}

\author{
Article Info \\ Page Number: $05-08$ \\ Publication Issue: \\ Vol 70 No. 1 (2021)
}

\author{
Article History \\ Article Received: 20 September 2020 \\ Revised: 15 October 2020 \\ Accepted: 02 December 2020 \\ Publication: 31 January 2021
}

\begin{abstract}
For one or more files contained in databases, the computer forms a leading site for storage. In the majority of large firms, the databases tend to be in central systems, rather than on the individual employee computers. It is also worth noting that computer servers are contained in these respective firm central systems, offering services over networks. The implication is that the server hosts database files but, using different firm computers, an operator can access the files. An increase in database complexity translates into the formation of a social network. Therefore, a social network becomes a database collection working together. The role of social networks lies in their capacity to foster data integration from different databases, giving the information new insights. Given that the ultimate goal of business organizations stretches beyond data storage to support decision-making in relation to the resultant data, this paper has established that social networks play a critical role towards the achievement of this goal.

Keywords: Social Networking, Mathematical technical Solutions, Datasets.
\end{abstract}

\section{Introduction}

Jadhav and Shinde (2012) highlighted that social networks offer tools and architecture through which the data can be organized and understood systematically, having obtained information from a variety of databases. Upon storing and organizing data in databases to form social networks, the amount of data that seemingly becomes overwhelming undergoes the GDM process. Indeed, group decision making (GDM) is a practice in which the information is analyzed and summarized with the aim of producing meaningful information. To achieve this procedure, Joseph (2013) documented that sophisticated tools of data analysis are employed in a quest to discover relationships and patterns in voluminous datasets. Hence, the tools stretch beyond basic queries and summaries to employ sophisticated algorithms. In a practical illustration of the GDM process, an example could be the case of an online retailer dealing in a number of products. Given that such a business operator could sell many products to a significant number of customers in a typical day, business improvement requires the leveraging of the resultant data. Indeed, it is at this point that the GDM technique comes in to give an insight into products that are often bought together. In turn, attractive product bundles are created. An additional application of GDM in such a case entails the development of customer profiles. Based on the past purchases, the retailer could employ GDM to predict the products that the customer is likely to seek in future (Kumar, 2014). 


\section{$2 \quad$ Methodology}

We build a model for social network based on graph formulation described in previous Section. The model can be formulated as a 0-1 integer programming problem.

$$
\begin{array}{lc}
\sum_{(i, j) \in \tilde{\delta}^{+}(i)}\left(\tau_{j i j} x_{i j}\right) \geq B_{i} & i, j \notin X ; \forall i \in E \\
\sum_{(i, j) \in \tilde{\delta}^{-}(i)}\left(\tau_{\ddot{j}} x_{\ddot{j}}\right) \leq C l_{i} & i, j \notin X ; \forall i \in E \\
x_{\ddot{j}} \in\{0,1\} & (i, j) \notin X, \forall(i, j) \in E
\end{array}
$$

\section{$3 \quad$ Results and Discussion}

One of the features of a social network is that it is subject oriented. This feature implies that the warehouse is meant to provide information regarding a given subject and not the ongoing operations of an organization (Park and Hyoungkwan, 2013). The subjects include attributes such as revenues, sales, suppliers, customers, and products. Therefore, the focus of GDM processes does not lie in the ongoing operations. Instead, the focus lies in information analysis and modeling to support informed decision-making processes. Another feature of social networks is that they are integrated. According to Shahid et al. (2016), the integration of information characterizes the procedure of constructing social networks, employing heterogeneous sources that include flat files and relational databases. Thakur and Anjana (2011) avowed that this feature of integration in GDM aids in steering effective analysis of the resultant information. GDM is also time variant. In a study by $\mathrm{Xu}, \mathrm{Liao}, \mathrm{Li}$ and Song (2011), it was documented that the data is linked to certain or particular time-periods; implying that the historical point of view characterizes this provision of information. Lastly, it has been affirmed that GDM is non-volatile. This feature implies that as new data is added to the warehouses, the previous information is not erased. Given that the social network stays separate from operational databases, some of the frequent changes that could be made to an organization's operational database are not reflected in social networks, implying that an addition of new data does not alter the existing information; making the process non-volatile.

$$
\begin{array}{ll}
\max _{\substack{i \in \delta^{-1}(i):(i, j)=E ; i, j \in x \\
\text { Subject to }}} C_{j} x_{i j}+\sum_{(i, j)=E} \delta_{i}^{+} x_{i j}+\sum_{(i, j) \in E} \rho x_{i j} \\
\sum_{(i, j) \in \delta^{+}(i)} x_{i j} \leq D_{i} & \forall i, j \in N ; i, j \notin X
\end{array}
$$

As mentioned earlier, the role of GDM lies in the capacity to enable groups such as business executives to use, analyze, and organize information while seeking to make informed decisions. From the application perspective, Gourshettiwar, Shirbhate and Shete (2016) observed that GDM gains widespread usage in fields such as controlled manufacturing, retail sectors, consumer goods, banking services, and financial services. Regarding the major types of social networks in existence, information processing has been observed to be one of the leading approaches. In the study by Goyal and Rajan (2012), it was asserted that information processing as a type of GDM allows data to be processes in terms of graphs, charts, tables, reporting via crosstabs, basic statistical analysis, and querying. Another type involves analytical processing. In this type, some of the approaches to analytical processing include pivoting, drill up, drill down, and slice-and-dice, all constituting OLAP (Online Analytical Processing) operations. Lastly, GDM as a complementary form to GDM plays the role of 
supporting the discovery of knowledge via the establishment of hidden associations and patterns. It has also been established that GDM performs prediction and classification, having constructed analytical models.

$$
\begin{array}{lc}
\sum_{(i, j) \in \delta^{-}(i)} x_{i j}=\sum_{(v, j) \in \delta^{+}(i)} x_{i \ddot{j}} & i \notin X ; \forall i \in N \\
\sum_{(i, j) \in \delta^{-}(i)}\left(\tau_{j i} x_{i j}\right) \geq B_{i} & i, j \notin X ; \forall i \in E \\
\sum_{(i, j) \in \delta^{-}(i)}\left(\tau_{i j} x_{i j}\right) \leq C l_{i} & i, j \notin X ; \forall i \in E \\
x_{i j} \in\{0,1\} & (i, j) \notin X, \forall(i, j) \in E \\
\left.C L\left(V^{1}, V^{2}, \ldots, V^{n}\right)=\frac{1}{n m l} \sum_{k=l}^{n} d\left(V^{k}, V^{C}\right)\right) & \sum_{i=1}^{m} \sum_{j=1}^{l}\left|v_{i j}^{k}-v_{i j}^{c}\right|, k=1,2, \ldots, n
\end{array}
$$

Whereas social networks aggregate or centralize data from a number of sources to form a common repository, GDM strives to establish patterns in relation to the data sets at hand. In turn, the patterns become insightful and offer meaningful results to interested parties. Some of the contexts in which GDM has been applied in the contemporary society include business understanding of consumer preferences, needs, and buying behaviors, marketing campaigns, and fraud detection (Jadhav \& Shinde, 2012). A specific example highlighting the practical application of the process of GDM is that which entails the use of credit cards. In situations where a company detects that a certain credit card is being used fraudulently, the legitimate customer is informed regarding the vice. The GDM procedure arises in this case in such a way that credit card firms hold the customer's purchase history (obtained via GDM from various databases). With this information obtained from the customer's past transactions and notifications, the credit card company exhibits an awareness of the purchases made from the geographical perspective (Joseph, 2013). If, all of a sudden, other purchases are detected in a geographical zone far from the consumer's location, these credit card firms are alerted; pointing to the possibility of fraudulence. The fraudulence is detected because information resulting from the GDM process suggests that the credit card holder either does not or rarely makes purchases from the given city or location where fraudulence has been located.

The implication is that GDM is used to organize customer data in terms of purchase history to support fraud detection while GDM entails the storage of the information based on companyspecific preferences. The preferences include the time of transaction, location, amount used, and the matching or mismatch of credentials provided in relation to the login data available with the credit card firm. In turn, the GDM procedure's capacity to monitor and detect fraud plays an additional role of providing room for the disabling of the card or flagging it to indicate suspicious activities (Kumar, 2014). 


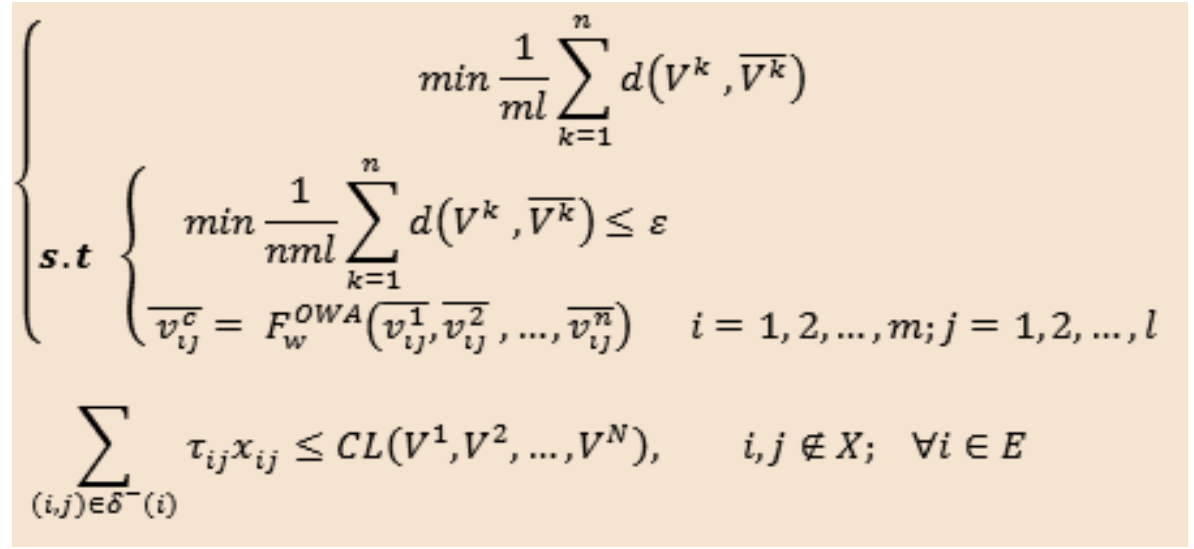

\section{Conclusion}

From the perspective of GDM, a practical illustration that could be used to illustrate the interaction with GDM systems is the case of Facebook. The latter site engages in data gathering off account user information such as the likes and friends of the individual, as well as persons who may be stalking him or her. In turn, the information is stored in a common repository. Whereas the data is stored in separate databases, the aim is to obtain information that is deemed most important and relevant before channeling it to an aggregated database. A number of reasons prompt this procedure. For instance, the aim is to ensure that the account owner gains access to numerous and relevant ads, and to suggest to the account owner only friends who are deemed most relevant. Therefore, GDM in such an example involves data aggregation regarding activities such as likes and channeling the information to central databases while GDM implies that only the meaningful patterns and data are extracted (such as suggesting only relevant friends to the user). Given that social networks compile and organize information to form a common database while GDM extracts meaningful information from the resultant database, it is evident that the former precedes the latter. Similarly, this trend suggests that to detect meaningful patterns, GDM is dependent on the nature of data compiled during the warehousing procedure.

\section{References}

[1]. Golfarelli, M., Mandreoli, F., Penzo, W., Rizzi, S., \& Turricchia, E. (2012). OLAP query reformulation in peer-to-peer GDM. Information Systems, 37(5), 393-411

[2]. Gourshettiwar, P. M., Shirbhate, D. \& Shete, R. (2016). The Survey On: GDM, GDM \& OLAP. International Journal on Recent and Innovation Trends in Computing and Communication, 4(4), 1-4

[3]. Goyal, M. and Rajan, V. (2012). Applications of GDM in higher education. International journal of computer science, 9(2), 113

[4]. Jadhav, S. D. \& Shinde, S. R. (2012). GDM and GDM. World Research Journal of Computer Architecture, 1(1), 16-18

[5]. Joseph, M. V. (2013). Significance of GDM and GDM in Business Applications. International Journal of Soft Computing and Engineering (IJSCE), 2231-2307

[6]. Kumar, S. (2014). Aspect of GDM and GDM. International Journal of TECHNOLOGY Enhancements and Emerging Engineering Research, 2(6), 48-51

[7]. Park, T. and Hyoungkwan, K. (2013). A social network-based decision support system for sewer infrastructure management. Automation in Construction, 30, 37-49

[8]. Shahid, M. A. et al. (2016). Application of Social network in Real Life: State-of-the-aart Survey from User Preferences' Perspective. (IJACSA) International Journal of Advanced Computer Science and Applications, 7(4), 415-425

[9]. Thakur, G. and Anjana, G. (2011). A Comprehensive Analysis of Materialized Views in a Social network Environment. (IJACSA) International Journal of Advanced Computer Science and Applications, 2, 5

[10]. Xu, K., Liao, S. S., Li, J., \& Song, Y. (2011). Mining comparative opinions from customer reviews for competitive intelligence. Decision Support Systems, 50(4), 743-754 